\title{
Diagnosis of breast cancer from mammograms by using fractal measures
}

\author{
Radu Dobrescu ${ }^{1}$, Loretta Ichim ${ }^{1,2,}$, , Daniela Crişan ${ }^{3}$ \\ ${ }^{1}$ Automatic Control and Industrial Informatics Department, Politechnic University of Bucharest, Bucharest, Romania \\ ${ }^{2}$ Stefan S. Nicolau Institute of Virology, Bucharest, Romania \\ ${ }^{3}$ Romanian-American University, Bucharest, Romania
}

\section{Email address:}

rd_dobrescu@yahoo.com (R. Dobrescu), loretta.ichim@aii.pub.ro (L. Ichim),dacrisan@yahoo.com (D. Crişan)

\section{To cite this article:}

Radu Dobrescu, Loretta Ichim, Daniela Crişan. Diagnosis of Breast Cancer from Mammograms by Using Fractal Measures. International Journal of Medical Imaging. Vol. 1, No. 2, 2013, pp. 32-38. doi: 10.11648/j.ijmi.20130102.14

\begin{abstract}
Breast cancer is the leading cause of cancer death among women. By the following research we report on a morphological study of 30 cases as seen in mammograms, trying to discriminate among benign and malignant tumors in order to develop new tools investigation in cancer diagnosis. From the contour of each mass, we computed the fractal dimension using box-counting algorithm and for each mammogram texture we computed the lacunarity. We found that the fractal dimension value is not sufficient to differentiate among benign and malignant cases, but it was really effective when it was combined with lacunarity. In conclusion, the results obtained showed that the fractal measure is an important tool for the diagnosis of breast cancer.
\end{abstract}

Keywords: Breast Cancer, Diagnosis, Fractal Dimension, Image Analysis, Lacunarity, Mammogram

\section{Introduction}

In most developed countries breast cancer is the leading cause of death among all cancers developed by middle-aged women. Any diagnosis tool helping to improve the sensitivity or the specificity of breast cancer would be highly valued. The usefulness of mammography in the symptomatic patient is undisputed; mammography is primarily used to demonstrate the presence of breast cancer and, specifically, to indicate the size and location of tumor [1].

There is also considerable evidence indicating the ability of mammography to detect cancer. In addition, randomized controlled trials of screening mammography have demonstrated a significant decline in breast cancer mortality among screened women of 50 years and older. In this sense, many automatic and semiautomatic techniques for cancer detection have been studied in the last years including topics such as segmentation, description, and classification.

Normal breasts have a wide variation in mammographic appearance. The pattern exhibited by a breast which is predominantly composed of fat can often be called normal if no disturbing pattern is found. There is not a qualitative or quantitative definition of what normality is, though most normal mammograms appear with regular and undisturbed ductal patterns.

Breast cancers usually appear with disturbed ductal structures. There are three major types of breast cancer: circumscribed/oval masses, spiculated lesions and microcalcifications. Malignant lesions generally have a more irregular shape than benign lesions. Circumscribed masses are compact and roughly elliptical. Radiolucent lesions with a halo or encapsulate are usually benign. High radiopaque lesion with irregular or ill-defined boundary should be considered with a high degree of suspicion. Spiculated lesions have a central tumor mass that is surrounded by a radiating pattern of linear spicules. Most spiculated lesions are malignant.

Microcalcifications appear as bright dot-spots on screening mammograms, usually in the form of clusters. These are calcium deposits from cell secretion and necrotic cellular debris. The shape and distribution of breast calcifications indicate malignancy. Benign microcalcifications are often smooth and sharply outlined and have high uniform density. Malignant microcalcifications usually appear in irregular shape and variably distributed [2].

The automated diagnosis in breast cancer holds great promise for large-scale use in the advanced cancer 
treatment, but it is not a straightforward task because a number of challenges have to be overcome. These challenges appear due to the complex nature of the breast tumors images (i.e., its variation in textural behavior and to the noise existence). Therefore the concern about the selection of efficient and enough features set to represent a certain tissue in the task of tissue-level property quantification was the target of many research efforts spent in the last two decades.

Several researchers have shown the potential of fractal analysis as a morphometric measure of the tumor's irregular structures, taking into consideration that:

1. Fractal geometry provides a general framework for the study of irregular sets, and has become popular in modeling these properties in texture image analysis and processing.

2. All the breast tissues structures have a statistical quality of roughness and statistical self-similarity at different scales.

3. The fractal dimension (FD) is an accurate measure of the roughness of a texture surface. Somehow, fractal dimension captures the notion of "how large a set is", it measures the rate of addition of structural detail with increasing magnification, scale or resolution.

4. The concept of multi-fractal has been used extensively for graphical simulation of natural phenomena, study of image textures and study of material surfaces.

Evidence of a breast tumor is usually indicated by the presence of a dense mass and/or a change in the texture or distortion in the mammogram. Consequently, the focus during diagnosis is on identifying such abnormal regions, as well as on classifying the type of mass or tumor that caused the abnormality. A typical benign mass is round and smooth with a well-defined (well-circumscribed) boundary, whereas a typical malignant tumor is spiculated and rough with a blurry boundary [3].

The breast is composed of a mixture of epithelial and fibro-glandular tissue together with fatty tissue, called parenchymal pattern. Fat is lucent and appears dark on the XRay image, while fibroglandular tissue appears to be dense and brighter. Mammographic parenchymal patterns are being increasingly used as intermediate markers in the studies investigating the etiology of breast cancer. During the last few years the researchers tried to develop a quantitative measure that might indicate the change of the tissue pattern or of the density in mammograms. This includes techniques of image texture analysis such as statistics of gray level distribution [4], Laws Energy [5], Wavelet [6], Gabor [7] and Fractal measures. Fractal measures are most frequently used in mammogram image analysis in application to mass detection [8, 9], tissue classification [10], microcalcification [11].

The main aim of this paper is to develop new investigation tools based on fractal analysis and lacunarity in order to increase the diagnosis accuracy in breast cancer, with no further investigation being needed.

\section{Related Works}

Numerous papers, starting with the seminal work of Landini and Rippin [12], have shown for the last 20 years that fractal geometry can be useful in the process of describing the pathological architecture of tumors. Fractal geometry has been known as a mathematical concept for many years and was introduced by B. Mandelbrot [13]. Its tools were successfully applied to characterize irregularly shaped and complex figures by a mathematical value wherever Euclidean geometry fails. One of the advantages of fractal analysis is the ability to quantify the irregularity and complexity of objects with a measurable value which is called the fractal dimension. In the past few years it has gained increasing attention in the frame of medical sciences, particularly in cardiology, pathology and radiology [14, 15] and has been applied in health conditions as well as in certain pathological conditions [16, 17]. During the past years fractal analysis has been applied also in tumor pathology to characterize irregular boundaries of tumors and their nuclei $[18,19]$. The fractal dimension is a measure of the complexity of a structure (a shape or a contour). In some sense, it is a measure of how complicated a structure is. All these studies emphasize the usefulness of fractal parameters in tumor pathology.

Fractal geometry has been intensively used in mammography for the diagnosis of breast cancer. In particular, the application of fractal geometry showed interesting results. There are many reports on the use of this geometry in mammography images [20-22].

In this field, the applicability of the fractal geometry is justified by the fact that micro-calcifications usually appear as a cluster of bright spots with variant size and shape embedded in an inhomogeneous background of breast tissue. The inhomogeneous background also exhibits the self-similarity property of typical fractal images such that a region of interest in digital mammograms containing the micro-calcifications clusters can be viewed as a fractal normal background superimposed by a non-fractal foreground (i.e. the micro-calcifications clusters) [23, 24].

Histopathology is the microscopic examination of body tissues and it is widely used for cancer diagnosis. In a traditional histopathologic investigation, pathologists visually examine body tissues using an optical or digital microscope and assess the deviations in the cell structures and/or the change in the distribution of the cells across the tissue under examination in order to determine the malignancy of tissues. Tumors are graded depending on the degree of malignancy. The grading of cancer is a significant challenge for pathologists because of uneven tumor growth and of their similar structural appearances within the grades. However, this judgment is subjective and often leads to considerable variability [25]. Computational image interpretation and classification methods may partially circumvent these limitations and improve the reliability of decisions resulting from pathologists diagnoses. 
Based on feature-extraction techniques histopathological image classification systems can be categorized into three classes:

(i) Class I: extracts the morphological features of cellular or subcellular components such as nuclei, lumen, and cytoplasm.

(ii) Class II: extracts the textural features by using feature descriptors such as filter banks, LBP, Haralick operator, fractal computation.

(iii) Class III: extracts both morphological and textural features.

Each method has different advantages and disadvantages. For example, the performance of class I methods is based on the segmentation accuracy of desired cellular or sub-cellular components. On the contrary, class II methods may overcome the limitation by observing the characteristics of the entire texture. However they may extract some undesired texture regions such as muscles or fiber.

The multifractal analysis-based classification and retrieval method was proposed by [26] to classify tissues of different body organs. They investigated the dissimilarities of the patterns of multifractal spectrums of three body organs, including the liver, lung, and artery. The authors argued that the distribution patterns of the multifractal spectrum can be utilized to distinguish between the tissue images of different body organs.

The use of fractal geometry to describe the texture of histopathological images can be found in [14, 27]. The fractal dimension of an object is a non-integer exponent that can be used to describe the complexity of a self similar structure. Multifractal analysis is a generalization of fractal analysis and it aims to describe natural structures (or images) as a spectrum of fractal dimensions. The method transforms a given texture from a gray-scale domain into a high-dimensional feature space by utilizing multifractal computation. A particular vector in the feature space contains the local behavior of the corresponding point in the gray-image and the information pertaining to its spatial distribution over the entire image.

\section{Materials and Methods}

\subsection{Patients}

Standard imaging protocols for mammary tumor have been used for this study. A set of 30 images from Fundeni Clinical Institute of Bucharest, collected between September 2006 and October 2008, was investigated, respectively 18 images showing benign tumors and 12 showing malign ones. A mammography is classified in a BI-RADS category (Breast Imagining Reporting Data System) from 0 to 6 [28]. The 1-3 categories signify that the probability to be a malignant tumor is very small; the 5 th category means that the probability to be a malignant tumor is very high, the 6th category known biopsy - proven malignancy and 0 category needs additional evaluation. In the 4th category the malignancy risk is $5-50 \%$ and, in this case, a biopsy is necessary. The tumor aspect is opaque with blurred edges. Thus, the information about malignity is concentrated in the tumor contour. The images in .jpg format with $1024 \times 1024$ pixels were converted in images .bmp, true color. For each image was traced by a radiologist the region of interest (FAR- Focus Attention Region) which was analyzed.

\subsection{Image Analyzes}

After image acquisition, a primary image processing (noise rejection, segmentation - in order to obtain binary image - and contour extraction) is necessary. In order to discriminate among healthy tissue and tumor mass we analyze each image pixel using specific criteria (similarity of the $3 \times 3$ pixel neighborhood average to the gray level of tumor mass center, pixel similarity to the health tissue gray level, pixel similarity to the tumor mass center gray level).

The diagnostic classification of the masses was based on biopsy. The contour of each mass was manually drawn by an expert radiologist specialized in mammography. The data set includes circumscribed and spiculated cases in both benign and malign categories. In the next figures we presented an example of mammography where the radiologist selected a risk area (Fig. $1 \mathrm{a}, \mathrm{b})$.

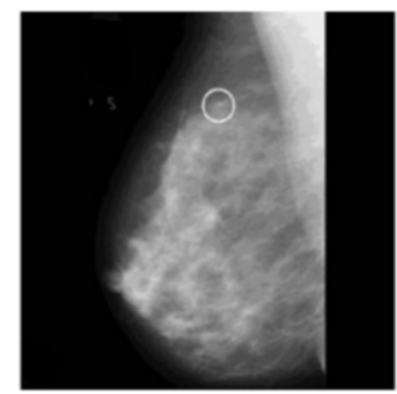

(a)

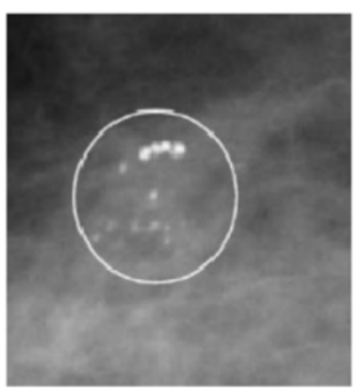

(b)
Figure 1. (a) The original mammogram; (b) the white circle indicates the suspicious region of interest (aria with microcalcifications).

\subsection{Fractal Dimension and Lacunarity Estimation}

The outline of each image was analyzed by estimating the fractal dimension. In brief, the fractal dimension of each outline was measured by the box-counting algorithm [29].

The box-counting method consists on partitioning the pattern or image space into square boxes of equal size and on counting the number of boxes that contain a part (at least one pixel) of the image. The process is repeated with partitioning of the image space into smaller and smaller squares. The log of the number of boxes counted is plotted against the log of the magnification index for each stage of partitioning, yielding a set of points on a line. The slope of the best-fitting straight line to the plot as above gives the FD of the pattern.

Lacunarity [30] is a counterpart to the fractal dimension that describes the texture of a fractal. Lacunarity is related 
to the size distribution of the holes. If a fractal has large gaps or holes, it has high lacunarity; on the other hand, if a fractal is almost translationally invariant it has low lacunarity. Lacunarity measures the distribution of gap sizes: low lacunarity geometric objects are homogeneous because all gap sizes are the same, whereas high lacunarity objects are heterogeneous. This measure supplements fractal dimensions in characterizing patterns extracted from digital images.

Lacunarity $(\lambda)$ is generally based on the pixel distribution for an image, that one can get from scans at different box sizes at different grid orientations. Basic number for lacunarity, $\lambda$, is $\lambda_{\varepsilon, \mathrm{g}}=(\sigma / \mu)_{\varepsilon, \mathrm{g}}^{2}$ where $\sigma$ is the standard deviation of the number of pixels that were in a box of size and $\mu$ is the mean for pixels per box at this size, $\varepsilon$, in a box count at this orientation, g. To put heterogeneity from one perspective and one series of grid sizes into an average, the mean $(\lambda)$ from all $\varepsilon$ sized boxes at a grid orientation, $g$, is calculated. We have used 16 to 48 pixel size boxes for each of four grid position on ROI (Region of Interest).

\section{Results and Discussion}

The algorithms for computing fractal dimensions were implemented in Microsoft Visual $\mathrm{C}++6.0$ software. For this purpose we used an original software package described in detail elsewhere [31]. The lacunarity was calculated using ImageJ [32] with Java plugin FracLac [33].

In Fig. 2 we have two cases of mammograms where the radiologist classified BI-RADS 2 for image (a) and classified BI-RADS 5 for image (b).

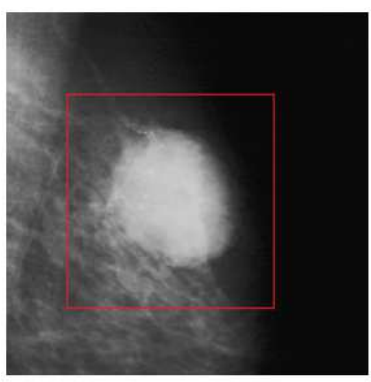

(a)

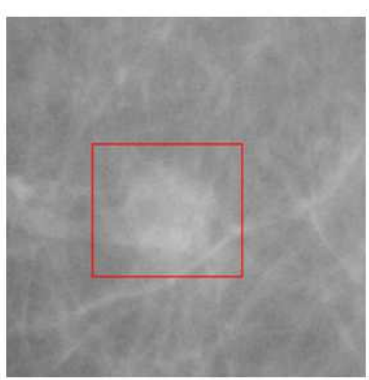

(b)
Figure 2. Examples of mammograms: (a) benign tumor $(1024 \times 1024)$; (b) malignant tumor $(1024 \times 1024)$.

For example we presented in Fig. 3 the contours of 6 benign cases and 6 malignant tumors having associated fractal dimension values from the total of 18 benign and 12 malignant images of tumors. Malignant tumors generally have higher FD because they are more ragged and spiculated than benign masses.

In Fig. 4 are shown the results of fractal analysis for benign and malignant tumors analyzed on contour of tumors using box-counting algorithm.

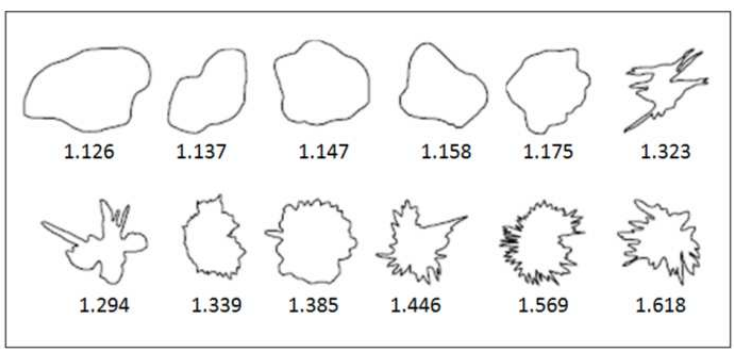

Figure 3. Contours of 6 benign masses (above) and 6 malignant tumors (below) " $F D$ values for benign tumors: 1.126, 1.137, 1.147, 1.158, 1.175, 1.323 and $F D$ values for malignant tumors: $1.294,1.339,1.385,1.446$, $1.569,1.618^{\prime \prime}$.

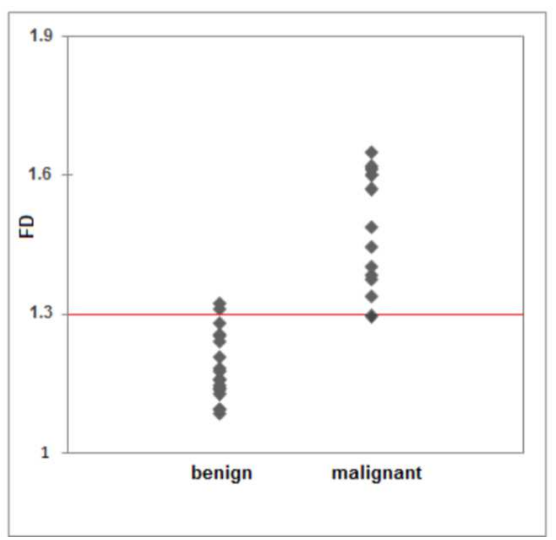

Figure 4. Fractal dimension values in benign and malignant tumors where establish the threshold 1.3 (red line).

Note that in Fig. 4, the last figure (at right) in the top row has the fractal dimension larger than the threshold 1.3, while the fractal dimension of the first figure (at left) in the bottom row is less than the threshold.

The experimental values of FD are presented in table 1 .

Table 1. Experimental values of $F D$

\begin{tabular}{lcccc}
\hline & Benign $(\mathbf{n}=\mathbf{1 8})$ & Malignant $(\mathbf{n}=\mathbf{1 2})$ \\
\hline FD values & $<1.3$ & $>1.3$ & $<1.3$ & $>1.3$ \\
Cases & 16 & 2 & 1 & 11 \\
Percentage & $89 \%$ & $11 \%$ & $8 \%$ & $92 \%$ \\
\hline
\end{tabular}

After the investigation of the tumor evolution, 18 cases were classified as benign and other 12 cases were classified as malignant. The percentage of correct diagnosis in the malignant case was approximately $92 \%$.

For this reason we have proposed to associate to the fractal dimension the lacunariy in order to better discriminate among malign and benign masses. Lacunarity is frequently assessed during fractal analysis.

Lacunarity explicitly characterize the spatial organization of an image and its composing sub-units, which are potentially useful in representing the tumor inner structure. From the anatomical point of view, the lacunarity helps to estimate the spatial heterogeneity of the lesions when the object complexity given by fractal dimension is not enough. 
In Fig. 5 are shown the results of lacunarity for benign and malign tumors analyzed on mammogram's image texture with the Image $\mathrm{J}$ software.

Note that in Fig. 5 the last figure (at right) in the top row has the lacunarity larger than the threshold 2, while the lacunarity of the first figure (at left) in the bottom row is less than the threshold. Table 2 shows the FD and lacunarity values and disease state the clinical case analyzed.

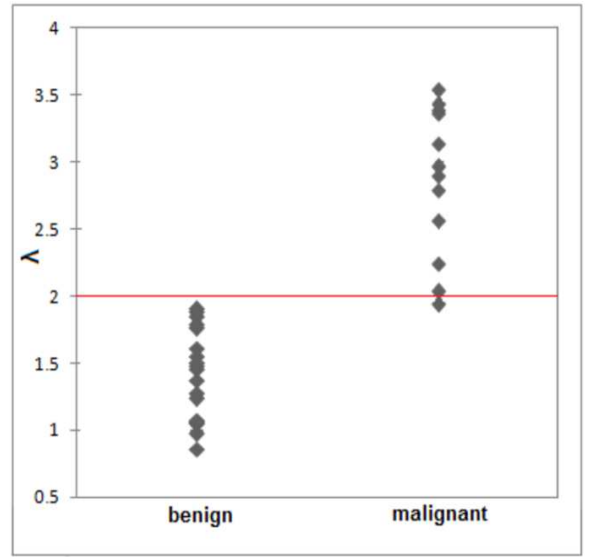

Figure 5. Lacunarity values in benign and malignant tumors where establish the threshold 2 (red line).

In table 2 it is observable that the three cases below or above (marked with + in table) the threshold of 1.3 in FD are different from the case in lacunarity.

Table 2. Clinical cases, FD and lacunarity values

\begin{tabular}{cccccc}
\hline $\begin{array}{c}\text { Case } \\
\text { ID }\end{array}$ & $\begin{array}{c}\text { Patient } \\
\text { ID }\end{array}$ & BI-RADS & $\begin{array}{c}\text { Disease } \\
\text { state }\end{array}$ & FD values & $\begin{array}{c}\text { Lacunarity } \\
\text { values }(\boldsymbol{\lambda})\end{array}$ \\
\hline 01 & P01 & 2 & $\mathrm{~B}$ & 1.085 & 0.858 \\
02 & $\mathrm{P} 02$ & 3 & $\mathrm{~B}$ & 1.241 & 1.604 \\
03 & $\mathrm{P} 03$ & 5 & $\mathrm{M}$ & 1.569 & 2.789 \\
04 & $\mathrm{P} 04$ & 4 & $\mathrm{M}$ & 1.375 & 2.030 \\
05 & $\mathrm{P} 05$ & 3 & $\mathrm{~B}$ & $1.323^{+}$ & 1.756 \\
06 & $\mathrm{P} 06$ & 6 & $\mathrm{M}$ & 1.618 & 3.382 \\
07 & $\mathrm{P} 07$ & 2 & $\mathrm{~B}$ & 1.147 & 0.971 \\
08 & $\mathrm{P} 08$ & 2 & $\mathrm{~B}$ & 1.157 & 1.372 \\
09 & $\mathrm{P} 09$ & 5 & $\mathrm{M}$ & 1.489 & 3.133 \\
10 & $\mathrm{P} 10$ & 3 & $\mathrm{~B}$ & 1.175 & 1.902 \\
11 & $\mathrm{P} 11$ & 5 & $\mathrm{M}$ & $1.294^{+}$ & 3.429 \\
12 & $\mathrm{P} 12$ & 2 & $\mathrm{~B}$ & 1.094 & 1.041 \\
13 & $\mathrm{P} 13$ & 3 & $\mathrm{~B}$ & 1.183 & 1.876 \\
14 & $\mathrm{P} 14$ & 6 & $\mathrm{M}$ & 1.601 & 3.540 \\
15 & $\mathrm{P} 15$ & 4 & $\mathrm{M}$ & 1.339 & 1.935 \\
16 & $\mathrm{P} 16$ & 5 & $\mathrm{M}$ & 1.446 & 2.900 \\
17 & $\mathrm{P} 17$ & 2 & $\mathrm{~B}$ & 1.158 & 1.052
\end{tabular}

\begin{tabular}{cccccc}
\hline $\begin{array}{c}\text { Case } \\
\text { ID }\end{array}$ & $\begin{array}{c}\text { Patient } \\
\text { ID }\end{array}$ & BI-RADS & $\begin{array}{c}\text { Disease } \\
\text { state }\end{array}$ & FD values & $\begin{array}{c}\text { Lacunarity } \\
\text { values }(\lambda)\end{array}$ \\
\hline 18 & P18 & 6 & M & 1.612 & 2.237 \\
19 & P19 & 5 & M & 1.402 & 2.960 \\
20 & P20 & 2 & B & 1.095 & 1.453 \\
21 & P21 & 2 & B & 1.126 & 1.070 \\
22 & P22 & 3 & B & $1.311^{+}$ & 1.502 \\
23 & P23 & 3 & B & 1.257 & 1.781 \\
24 & P24 & 2 & B & 1.137 & 1.231 \\
25 & P25 & 3 & B & 1.280 & 1.476 \\
26 & P26 & 4 & M & 1.385 & 2.562 \\
27 & P27 & 3 & B & 1.208 & 1.546 \\
28 & P28 & 6 & M & 1.651 & 3.359 \\
29 & P29 & 2 & B & 1.139 & 1.277 \\
30 & P30 & 3 & B & 1.254 & 1.843 \\
\hline
\end{tabular}

${ }^{+}$three cases of FD values below or above the threshold of 1.3

The experimental values of lacunarity are presented in table 3 .

Table 3. Experimental values of lacunarity

\begin{tabular}{ccccc}
\hline & \multicolumn{2}{c}{ Benign $(\mathbf{n}=\mathbf{1 8})$} & \multicolumn{2}{c}{ Malignant $(\mathbf{n}=\mathbf{1 2})$} \\
\hline Lacunarity values $(\lambda)$ & $<2$ & $>2$ & $<2$ & $>2$ \\
Cases & 18 & 0 & 1 & 11 \\
Percentage & $100 \%$ & $0 \%$ & $8 \%$ & $92 \%$ \\
\hline
\end{tabular}

Significant differences were found between DF values in benign and malignant tumors using $\mathrm{t}$-test $(\mathrm{t}=8.126, \mathrm{p}=$ $0.01)$ and between lacunarity values $(t=8.814, p=0.01)$ respectively.

\section{Conclusions and Future Works}

This study identified two best predictors of breast cancer risk among the computer-based quantitative measures of mammographic features.

In this application using fractal dimension we can distinguish healthy from unhealthy subjects. Fractal analysis is often brought to the evaluation of the FD that allows having a global description of the lack of homogeneities in the image. The applicability of this geometry in image analysis comes from the fact that the depicted objects are discontinuous, complex, and fragmented. The significance and the advantage of this geometry compared to classical processing methods, is due to way in which the non-regularities are assumed and identified.

The results show that there is no situation wherein a tumor (benign or malign) to be placed outside of both thresholds. Among 30 cases, 27 (90\%) were correctly classified, and in three cases the decision was related to 
"insufficient information to formulate a diagnosis". The fractal dimension values and lacunarity obtained are consistent with those presented in literature [34].

Fractal dimension might be a helpful adjunctive technique to distinguish among benign and malign cases of mammary tumors. Is has been proven to be really effective when it was combined with lacunarity in the classification process of breast masses and in the diagnosis of breast cancer. We found a good association between FD, a measure of the contour of a tumor and lacunarity, a measure of texture in breast cancer. When FD was combined with lacunarity, the classification accuracy was improved. Combinations of shape factors and texture measures would cetrainly be more effective in the classification of breast masses than any type of features on its own. These results have also a good correlation with the visual inspection done by the pathologist.

One of the main limitations of the study is the relatively small number of breast cancer cases acquired for a certain period of time. Aiming more relevant statistics, we presently work to extend the database with medical images of the patients with breast carcinoma.

\section{Acknowledgements}

This work was supported by the FP7 project ERRIC Empowering Romanian Research on Intelligent Information Technologies, REGPOT-2010-1 No. 264207.

\section{References}

[1] E. B. Mendelson, "Current status of breast: Categorical course in diagnostic radiology physics," Physical Aspects of Breast Considerations, pp. 295-309, 1999.

[2] L. Tabar, and P. Dean, Teaching atlas of mammography, New York: Time. 3rd ed., 2001.

[3] R. M. Rangayyan, and T. M. Nguyen, "Fractal Analysis of Contours of Breast Masses in Mammograms," J. Digit. Imaging, vol. 20, pp. 223-237, Sep. 2007.

[4] H. Li, "Computer texture analysis of mammographic parenchymal patterns of digitized mammograms," International Congress Serie, vol. 1268, pp. 878-881, 2004.

[5] R. Gupta, and P. E. Undrill, "The use of texture analysis to delineate suspicious masses in mammography," Phys. Med. Biol., vol. 40, pp. 835-855, May 1995.

[6] C. M. Kocur, "Using neural networks to select wavelet features for breast cancer diagnosis," IEEE Eng. Med. Biol. Mag., vol. 15, pp. 95-102, 1996.

[7] R. J. Ferrari, "Analysis of asymmetry in mammograms via directional filtering with Gabor wavelets," IEEE Trans. Med. Imaging, vol. 20, pp. 953-964, Sep. 2001.

[8] M. E. Mavroforakis, "Mammographic masses characterization based on localized texture and dataset fractal analysis using linear, neural and support vector machine classifiers," Artif. Intell. Med., vol. 37, pp. 145-162, 2006.
[9] G. T. Tourassi, "A study on the computerized fractal analysis of architectural distortion in screening mammograms," Phys. Med. Biol., vol. 51, pp. 1299-1312, Mar. 2006.

[10] F. Georgsson, S. Jansson, and C. Olsen, "Fractal analysis of mammogram," Lecture Notes in Computer Science, vol. 4522, pp. 92-101, 2007.

[11] K. Yamada, "Quantitative expression of microcalcification distribution in mammograph by using fractal dimension," in Singapore ICCS/ISITA Communication on the Move, pp. 92-96, 1992.

[12] G. Landini, and J. W. Rippin, "Fractal dimensions of the epithelial-connective tissue interfaces in premalignant and malignant epithelial lesions of the floor of the mouth," Anal. Quant. Cytol. Histol., vol. 15, pp. 144-149, Apr. 1993.

[13] B. B. Mandelbrot, The fractal geometry of nature, Freeman: New York, 1983.

[14] S. S. Cross, "Fractals in pathology," J. Pathol., vol. 182, pp. 1-8, May 1997.

[15] G. A. Losa, and T. F. Nonnenmacher, "Self-similarity and fractal irregularity in pathologic tissues," Mod. Pathol., vol. 9, pp. 174-182, Mar. 1996.

[16] G. Landini, G. P. Misson, and P. I. Murray, "Fractal analysis of the normal human retinal fluorescein angiogram," Curr. Eye. Res., vol. 12, pp. 23-27, Jan. 1993.

[17] G. A. Losa, G. Baumann, and T. F. Nonnenmacher, "Fractal dimension of pericellular membranes in human lymphocytes and lymphoblastic leukemia cells," Pathol. Res. Pract., vol. 18, pp. 680-686, Jun. 1992.

[18] G. Landini, and J. W. Rippin, "Quantification of nuclear pleomorphism using an asymptotic fractal model," Anal. Quant. Cytol. Histol., vol. 18, pp. 167-176, Apr. 1996.

[19] S. S. Cross, J. P. Bury, P. B. Silcocks, T. J. Stephenson, and D. W. K. Cotton, "Fractal geometric analysis of colorectal polyps,” J. Pathol., vol. 172, pp. 317-323, Apr. 1994.

[20] H. Li, M. L. Giger, O. I. Olopade, and L. Lan, "Fractal analysis of mammographic parenchymal patterns in breast cancer risk assessment," Acad. Radiol., vol. 14, pp. 513-521, May 2007.

[21] T. Nguyen, and R. Rangayyan, "Shape Analysis of Breast Masses in Mammograms via the Fractal Dimension," in Proceedings of the 29th Annual International Conference of the IEEE Engineering in Medecine and Biology Society, pp. 3210-3213, 2005.

[22] N. Tanki, K. Murase, and M. Nagao, "A new parameter enhancing breast cancer detection in computer-aided diagnosis of X-ray mammograms," Igaku Butsuri, vol. 26, pp. 207-215, 2006

[23] Y. Huang, and S. Yu, "Recognition of micro-calcifications in digital mammograms based on Markov random fiels and deterministic fractal modeling," in Proceedings of the 29th Annual International Conference of the IEEE Engineering in Medicine and Biology Society, pp. 3922-3925, 2007.

[24] P. Kestener, J. Lina, P. Saint-Jean, and A. Arneodo, "Wavelet-based multifractal formalism to assist in diagnosis in digitized mammograms," Image Analysis and Stereology, vol. 20 , pp. $169-175,2004$. 
[25] L. E. George, and K. H. Sager, "Breast cancer diagnosis using multi-fractal dimension spectra," in Proceedings of the IEEE International Conference on Signal Processing and Communications, pp. 592-595, 2007.

[26] A. Hemsley, and R. Mukundan, "Multifractal measures for tissue image classification and retrieval," In Proceedings of the 11th IEEE International Symposium on Multimedia, pp. 618-623, 2009.

[27] T. Mattfeldt, "Spatial pattern analysis using chaos theory: A nonlinear deterministic approach to the histological texture of tumours," in Fractals in Biology and Medicine, vol. II, G. A. Losa, D. Merlini, T. F. Nonnenmacher, E. R. Weibel Eds. Basel, Boston, Berlin: Birkhäuser, pp. 50-72, 1997.

[28] American College of Radiology, Breast imaging reporting and data system atlas (BI-RADS Atlas), 2003.

[29] G. Landini, and J. W. Rippin, "How important is tumor shape? Quantification of the epithelial-connective tissue interface in oral lesions using local connected fractal dimension analysis," J. Pathol., vol. 179, pp. 210-217, Jun. 1996.

[30] G. Karemore, and M. Nielsen, "Fractal dimension and lacunarity analysis of mammographic patterns in assessing breast cancer risk related to HRT treated population: a longitudinal and cross-sectional study," in Proceedings of the SPIE, vol. 7260, pp. 72602F 1-9, 2009.

[31] D. Crişan, Image processing using fractal techniques. Ph. dissertation, Automatic Control and Industrial Informatics Dept., Politehnica University of Bucharest, Romania, 2005.

[32] W. S. Rasband, ImageJ, U. S. National Institutes of Health, Bethesda, Maryland, USA, http://rsb.info.nih.gov/ij/, 1997-2005.

[33] A. Karperien, FracLac for ImageJ, version 2.5, 1999-2007.

[34] R. Dobrescu, and C. Vasilescu Eds., Interdisciplinary applications of fractal and chaos theory, Romanian Academy Printing House: Bucharest, 2004. 